\title{
A Note on the Chevalley-Warning Theorems
}

\author{
D.R. Heath-Brown \\ Mathematical Institute, Oxford \\ In memory of Anatolii Karatsuba
}

\section{Introduction}

The most widely known result of Chevalley-Warning type states that if one has a polynomial over a finite field of characteristic $p$, and the number of variables exceeds the degree, then the number of zeros is a multiple of $p$. In particular, if the polynomial is homogeneous, there is at least one non-trivial zero.

There are a number of related results in the literature. Generally, let $\mathbb{F}_{q}$ be a finite field, and let $\mathbf{f}=\left(f_{1}(\mathbf{x}), \ldots, f_{r}(\mathbf{x})\right)$ be an $r$-tuple of polynomials $f_{i}\left(x_{1}, \ldots, x_{n}\right) \in \mathbb{F}_{q}\left[x_{1}, \ldots, x_{n}\right]$. Let $d_{i}$ be the total degree of $f_{i}$, and write $d=d_{1}+\ldots+d_{r}$. For any subset $S \subseteq \mathbb{F}_{q}^{n}$ we put

$$
z(\mathbf{f} ; S)=z(S):=\{\mathbf{x} \in S: \mathbf{f}(\mathbf{x})=0\}
$$

and

$$
\mathcal{N}(\mathbf{f} ; S)=\mathcal{N}(S):=\# z(\mathbf{f} ; S)
$$

Then the original 1935 result of Chevalley [2] stated that if $n>d$ and $z\left(\mathbb{F}_{q}^{n}\right)$ is non-empty, then it contains at least 2 points. Immediately afterwards it was shown by Warning [3] that if $n>d$ then

$$
p \mid \mathcal{N}\left(\mathbb{F}_{q}^{n}\right)
$$

where $p$ is the characteristic of $\mathbb{F}_{q}$. In fact this followed from a trivial rearrangement of Chevalley's argument. In the same paper Warning proved that if $n \geq d$, and if $H_{1}$ and $H_{2}$ are parallel affine hyperplanes in $\mathbb{F}_{q}^{n}$ then

$$
\mathcal{N}\left(H_{1}\right) \equiv \mathcal{N}\left(H_{2}\right)(\bmod p)
$$

As a corollary he deduced that if $z\left(\mathbb{F}_{q}^{n}\right)$ is non-empty then

$$
\mathcal{N}\left(\mathbb{F}_{q}^{n}\right) \geq q^{n-d} \quad(n>d)
$$


Later, in 1964, Ax [1] strengthened (1) by showing that indeed one has

$$
q \mid \mathcal{N}\left(\mathbb{F}_{q}^{n}\right) \quad(n>d)
$$

When $n$ is large compared with $d$ there are further improvements possible, as was shown by Katz [4] for example. These sharpenings of (44) lead to improvements of all the results we will establish. However we wish to focus here on the situation in which $n$ is not much larger than $d$, and so will be content with using (41).

Our first result shows that there is a strengthening of (2) corresponding to Ax's improvement of (11). To stress that the affine linear spaces we encounter are not necessarily vector subspaces of $\mathbb{F}_{q}^{n}$ we will work over $\mathbb{A}^{n}\left(\mathbb{F}_{q}\right)$, but of course the sets $z\left(\mathbb{A}^{n}\left(\mathbb{F}_{q}\right)\right)$ and $z\left(\mathbb{F}_{q}^{n}\right)$ are the same.

Theorem 1 With the notation above, we have

$$
\mathcal{N}\left(L_{1}\right) \equiv \mathcal{N}\left(L_{2}\right)(\bmod q)
$$

for any two parallel linear spaces $L_{1}, L_{2} \subseteq \mathbb{A}^{n}\left(\mathbb{F}_{q}\right)$ of dimension d or more.

Indeed our argument shows how one may deduce (5) from (44).

We next look at improvements to (3) . If $K=\mathbb{F}_{q^{k}}$ has field basis $\omega_{1}, \ldots, \omega_{k}$ over $F=\mathbb{F}_{q}$ we set

$$
N_{k}\left(x_{1}, \ldots, x_{k}\right):=N_{K / F}\left(x_{1} \omega_{1}+\ldots+x_{k} \omega_{k}\right) .
$$

This produces a form of degree $k$ in $k$ variables, with the property that $N_{k}\left(x_{1}, \ldots, x_{k}\right)=0$ with variables $x_{i} \in F_{q}$ only when the $x_{i}$ are all zero. In particular, if $\mathbf{f}$ consists of a single polynomial $f_{1}=N_{d}$ then $z\left(\mathbf{f} ; \mathbb{A}^{n}\left(\mathbb{F}_{q}\right)\right)$ will be a linear space of dimension $n-d$, and we will have equality in (3). In contrast we have the following results.

Theorem 2 Suppose that $n>d$ and that $z\left(\mathbb{A}^{n}\left(\mathbb{F}_{q}\right)\right)$ is non-empty, and is not a linear subspace of $\mathbb{A}^{n}\left(\mathbb{F}_{q}\right)$. Then

(i) For any $q$ we have $\mathcal{N}\left(\mathbb{A}^{n}\left(\mathbb{F}_{q}\right)\right)>q^{n-d}$;

(ii) If $q \geq 4$ we have $\mathcal{N}\left(\mathbb{A}^{n}\left(\mathbb{F}_{q}\right)\right) \geq 2 q^{n-d}$; and

(iii) For any $q$ we have $\mathcal{N}\left(\mathbb{A}^{n}\left(\mathbb{F}_{q}\right)\right) \geq q^{n+1-d} /(n+2-d)$ providing that the polynomials $\mathbf{f}$ are homogeneous. 
Our proof of this will use only combinatorial facts about $\mathbb{A}^{n}\left(\mathbb{F}_{q}\right)$, along with (51) and its consequence (44). Thus the analogues of these results would be true if one replaced $z\left(\mathbb{A}^{n}\left(\mathbb{F}_{q}\right)\right)$ by any set with the property (5) for every pair of parallel $d$-dimensional linear spaces $\Lambda_{1}, \Lambda_{2} \subseteq \mathbb{A}^{n}\left(\mathbb{F}_{q}\right)$. In particular we see that part (ii) is best possible in this sense, since (5) would hold if $z\left(\mathbb{A}^{n}\left(\mathbb{F}_{q}\right)\right)$ were composed of two parallel linear spaces of dimension $n-d$.

It may be instructive to examine two simple examples at this point. The first shows that one cannot hope to improve part (iii) of Theorem 2 to say that $\mathcal{N}\left(\mathbb{A}^{n}\left(\mathbb{F}_{q}\right)\right) \geq q^{n+1-d}$. Here we take $\mathbf{f}$ to consist of the single polynomial $Q\left(x_{1}, \ldots, x_{4}\right)$, where

$$
Q\left(x_{1}, x_{2}, x_{3}, x_{4}\right)=x_{1} x_{2}+x_{3}^{2}+x_{3} x_{4}+c x_{4}^{2}
$$

with the coefficient $c \in \mathbb{F}_{q}$ chosen so that $x_{3}^{3}+x_{3} x_{4}+c x_{4}^{2}$ does not factor over $\mathbb{F}_{q}$. Then $Q=0$ has precisely $q^{3}-q^{2}+q$ solutions $\left(x_{1}, \ldots, x_{4}\right) \in \mathbb{F}_{q}^{4}$, and since $d=2$ in this case we have

$$
\mathcal{N}\left(\mathbf{f} ; \mathbb{A}^{n}\left(\mathbb{F}_{q}\right)\right)=q^{n-4}\left(q^{3}-q^{2}+q\right)=q^{n+1-d}\left(1-q^{-1}+q^{-2}\right)<q^{n+1-d} .
$$

Indeed this also shows that part (ii) of the theorem cannot be extended to $q=2$.

Our second example shows that when $\mathbf{f}$ consists of a single polynomial $f_{1}$, it is possible for $z\left(\mathbb{A}^{n}\left(\mathbb{F}_{q}\right)\right)$ to be a linear space of dimension $n-k$ even though $f_{1}$ does not split into linear factors. For this we take $n=4$ and choose a field basis $1, \alpha$ for $\mathbb{F}_{q^{2}}$ over $\mathbb{F}_{q}$. Let $N$ and $N^{\prime}$ denote norm forms for $\mathbb{F}_{q^{4}}$ over $\mathbb{F}_{q^{2}}$ and $\mathbb{F}_{q^{2}}$ over $\mathbb{F}_{q}$ respectively, using the basis $1, \alpha$ in the latter case. Let $\sigma$ be the nontrivial automorphism of $\mathbb{F}_{q^{2}}$ over $\mathbb{F}_{q}$. Then we may write $N(\mathbf{x})=Q_{1}(\mathbf{x})+\alpha Q_{2}(\mathbf{x})$ for certain quadratic forms $Q_{1}, Q_{2}$ over $\mathbb{F}_{q}$. It is clear that we may use a linear change of variables over $\overline{\mathbb{F}_{q}}$ to write $Q_{1}+\alpha Q_{2}=Y_{1} Y_{2}$ and $Q_{1}+\alpha^{\sigma} Q_{2}=Y_{3} Y_{4}$ with independent variables $Y_{1}, \ldots, Y_{4}$. It follows that if we take $\beta \in \mathbb{F}_{q^{2}} \backslash \mathbb{F}_{q}$ different from $\alpha$ and $\alpha^{\sigma}$ then $Q_{1}+\beta Q_{2}=$ will have rank 4 . Such a $\beta$ certainly exists, except when $q=2$. We now see that the polynomial $f(\mathbf{x})=\left(Q_{1}(\mathbf{x})+\beta Q_{2}(\mathbf{x})\right)\left(Q_{1}(\mathbf{x})+\beta Q_{2}(\mathbf{x})\right)^{\sigma}$ is defined over $\mathbb{F}_{q}$ and does not split into linear factors. None the less we have $f=0$ only for $\mathbf{x}=0$.

Finally we will investigate the structure of $z\left(\mathbf{f} ; \mathbb{A}^{n}\left(\mathbb{F}_{q}\right)\right)$ geometrically.

Theorem 3 Given $n$ and $d$ there is a constant $C(n, d)$ as follows. For any set of polynomials $\mathbf{f}$ whose degrees total d there is a corresponding set of absolutely irreducible varieties $V_{1}, \ldots, V_{m}$ defined over $\mathbb{F}_{q}$ such that $z\left(\mathbf{f} ; \mathbb{A}^{n}\left(\mathbb{F}_{q}\right)\right)$ is precisely the set of $\mathbb{F}_{q}$-points in $V_{1} \cup \ldots \cup V_{m}$. Moreover each variety $V_{i}$ has degree at most $C(n, d)$, and $m \leq C(n, d)$. 
The Lang-Weil bound [5] now gives us the following immediate corollary.

Corollary Suppose that the largest dimension among the varieties $V_{i}$ is $D$, and that this occurs $k$ times. Then, enlarging $C(n, d)$ if necessary, we have

$$
\left|\mathcal{N}\left(\mathbf{f} ; \mathbb{A}^{n}\left(\mathbb{F}_{q}\right)\right)-k q^{D}\right| \leq C(n, d) q^{D-1 / 2} .
$$

Thus if $q \gg_{n, d} 1$ we have $D \geq n-d$, and indeed if $z\left(\mathbf{f} ; \mathbb{A}^{n}\left(\mathbb{F}_{q}\right)\right)$ is not a linear space of dimension $n-d$ then $D \geq n+1-d$. In the latter case we have

$$
\mathcal{N}\left(\mathbf{f} ; \mathbb{A}^{n}\left(\mathbb{F}_{q}\right)\right) \geq q^{n+1-d}-C(n, d) q^{n+1 / 2-d}
$$

for $q \gg_{n, d} 1$.

One would conjecture that $D \geq n-d$ irrespective of the size of $q$, and it would be of interest to have a direct geometrical proof of this. Of course, the lower bounds on $D$ follow on comparing (7) with (3) and part (iii) of Theorem 2 .

Acknowledgement This research was begun while the author was visiting the Institute for Advanced Study, in Princeton. The hospitality and financial support of the institute is gratefully acknowledged.

Thanks are also due to the anonymous referee for his careful reading of the original version of this paper, which resulted in the correction of two errors.

Finally, we would like to record our thanks to Professor David Leep, who pointed out a difficulty with our original treatment of Theorem 1.

\section{Proofs}

We now present our proof of Theorem 1. In view of (4) the result is trivial if $L_{1}$ and $L_{2}$ have dimension strictly greater than $d$, since we would have $q \mid \mathcal{N}\left(L_{1}\right)$ and $q \mid \mathcal{N}\left(L_{2}\right)$ in this case. We therefore suppose that $L_{1}$ and $L_{2}$ have dimension equal to $d$. Let $L=<\underline{\mathrm{e}}_{1}, \ldots, \underline{\mathrm{e}}_{d}>$ be the linear space parallel to $L_{1}$ and $L_{2}$ but passing through the origin, and let $L_{j}=L+\underline{\mathrm{c}}_{j}$ for $j=1$ and 2. Then $\mathcal{N}\left(\underline{f} ; L_{j}\right)=\mathcal{N}\left(\underline{\mathrm{g}}^{(j)} ; \mathbb{A}^{d}\left(\mathbb{F}_{q}\right)\right)$, where

$$
g_{i}^{(j)}\left(y_{1}, \ldots, y_{d}\right)=f_{i}\left(\sum_{k=1}^{d} y_{k} \underline{\mathrm{e}}_{k}+\underline{\mathrm{c}}_{j}\right) \quad(j=1,2,1 \leq i \leq r) .
$$

It could happen that the terms of degree $d_{i}$ in $g_{i}^{(1)}$ all vanish, but this happens if and only if the corresponding terms in $g_{i}^{(2)}$ also vanish. In this situation the 
total degree of each of the systems $\left(g_{1}^{(1)}, \ldots, g_{r}^{(1)}\right)$ and $\left(g_{1}^{(2)}, \ldots, g_{r}^{(2)}\right)$ will be strictly less than $d$, so that $q \mid \mathcal{N}\left(L_{1}\right)$ and $q \mid \mathcal{N}\left(L_{2}\right)$, by (4). It follows that we may assume that the leading homogeneous parts of $g_{i}^{(1)}$ and $g_{i}^{(2)}$, both have degree $d_{i}$. Indeed we then see that these leading homogeneous parts are the same.

Given a polynomial $f\left(x_{1}, \ldots, x_{n}\right)$ of total degree $e$ there are two reasonable ways of associating a form to it. One may take $f_{-}\left(x_{1}, \ldots, x_{n}\right)$ to be the homogeneous part of degree $e$, or one may define

$$
f_{+}\left(x_{0}, \ldots, x_{n}\right)=x_{0}^{e} f\left(x_{1} / x_{0}, \ldots, x_{n} / x_{0}\right) .
$$

For a system $\mathbf{f}$ we define $\mathbf{f}_{-}$and $\mathbf{f}_{+}$by the above processes, using degree $e=d_{i}$ for each polynomial $f_{i}$. Clearly each zero of $\mathbf{f}$ produces exactly $q-1$ zeros of $\mathbf{f}_{+}$with $x_{0} \neq 0$; and the zeros of $\mathbf{f}_{+}$with $x_{0}=0$ correspond precisely to the zeros of $\mathbf{f}_{-}$. Thus

$$
\mathcal{N}\left(\mathbf{f}_{+} ; \mathbb{F}_{q}^{n+1}\right)=(q-1) \mathcal{N}\left(\mathbf{f} ; \mathbb{F}_{q}^{n}\right)+\mathcal{N}\left(\mathbf{f}_{-} ; \mathbb{F}_{q}^{n}\right)
$$

In particular, if $n \geq d$ then (4) yields $q \mid \mathcal{N}\left(\mathbf{f}_{+} ; \mathbb{F}_{q}^{n+1}\right)$ and hence

$$
\mathcal{N}\left(\mathbf{f} ; \mathbb{F}_{q}^{n}\right) \equiv \mathcal{N}\left(\mathbf{f}_{-} ; \mathbb{F}_{q}^{n}\right)(\bmod q)
$$

We therefore see that the value of $\mathcal{N}\left(\mathbf{f} ; \mathbb{F}_{q}^{n}\right)$ modulo $q$ only depends on the leading homogeneous parts of the polynomials $f_{1}, \ldots, f_{r}$.

Now suppose we have parallel $d$-dimensional affine linear spaces $L_{1}$ and $L_{2}$ in $\mathbb{F}_{q}^{n}$. We represent the restriction of the polynomials $\mathbf{f}$ to $L_{1}$ by a set of polynomials $\left(g_{1}^{(1)}, \ldots, g_{r}^{(1)}\right)$ in $d$ variables, and similarly for $L_{2}$. As discussed above, the leading homogeneous parts of $g_{i}^{(1)}$ and $g_{i}^{(2)}$ will be the same, and we are therefore led to Theorem 1 .

Our proof of Theorem 2 is based on the following two lemmas.

Lemma 1 Let $L_{0} \subseteq \mathbb{A}^{n}\left(\mathbb{F}_{q}\right)$ be a linear space. Choose a linear space $L$ of maximal dimension, $k$ say, such that $L \supseteq L_{0}$ and $\mathcal{N}(L)=\mathcal{N}\left(L_{0}\right)$. Suppose $L^{\prime} \supset L$ is a linear space of dimension $k+1$ such that $\mathcal{N}\left(L^{\prime}\right)$ is minimal. Then

$$
\mathcal{N}\left(\mathbb{A}^{n}\left(\mathbb{F}_{q}\right)\right) \geq \mathcal{N}(L)+\frac{q^{n-k}-1}{q-1}\left(\mathcal{N}\left(L^{\prime}\right)-\mathcal{N}(L)\right)
$$

Lemma 2 Let $S \subseteq \mathbb{A}^{t}\left(\mathbb{F}_{q}\right)$ be a set containing $t+1$ points in general position. Then

(i) If $q=2$, and if there is no 2-plane $L \subseteq \mathbb{A}^{t}\left(\mathbb{F}_{q}\right)$ meeting $S$ in exactly 3 points, then $S=\mathbb{A}^{t}\left(\mathbb{F}_{q}\right)$. 
(ii) If $q \geq 3$, and if $\ell \subseteq S$ for every line $\ell$ meeting $S$ in at least two points, then $S=\mathbb{A}^{t}\left(\mathbb{F}_{q}\right)$.

(iii) If $q \geq 4$, and if $\#(S \cap \ell) \geq q-1$ for every line $\ell$ meeting $S$ in at least two points, then $\mathbb{A}^{t}\left(\mathbb{F}_{q}\right) \backslash S$ is contained in a hyperplane.

(iv) If $m \geq 2$ is an integer, and if $\#(S \cap \ell) \geq m+1$ for every line $\ell$ meeting $S$ in at least two points, then

$$
\# S \geq \frac{m^{t+1}-1}{m-1}
$$

To deduce Theorem 2 we assume that $z\left(\mathbb{A}^{n}\left(\mathbb{F}_{q}\right)\right)$ contains a maximal set of $t \geq n+1-d$ points in general position. We take $\mathbb{A}^{t}\left(\mathbb{F}_{q}\right)$ to be the space spanned by these points and apply Lemma 2 to the set $S$ consisting of zeros of $\mathbf{f}$ lying in $\mathbb{A}^{t}\left(\mathbb{F}_{q}\right)$. We will give the details required for the different parts of Theorem 2 in due course, but the general strategy is as follows. Lemma 2 will either provide a suitable lower bound for $\mathcal{N}\left(\mathbb{A}^{n}\left(\mathbb{F}_{q}\right)\right)$, or produce a linear space $L_{0}$ to which we will apply Lemma 1. This linear space will have dimension two in the case of part (i) of Lemma 2, or dimension one otherwise. We will have arranged that $\mathcal{N}\left(L_{0}\right) \leq q-1$, and since $\mathcal{N}(L)=\mathcal{N}\left(L_{0}\right)$ in Lemma 11 the dimension $k$ of $L$ can be at most $d$, for otherwise (5) will give us a contradiction. If $k \leq d-2$ then (8) implies that

$$
\mathcal{N}\left(\mathbb{A}^{n}\left(\mathbb{F}_{q}\right)\right) \geq \frac{q^{n-k}-1}{q-1} \geq \frac{q^{n+2-d}-1}{q-1} \geq^{n+1-d},
$$

which is a satisfactory lower bound. Thus we may assume that either $L$ or $L^{\prime}$ has dimension $d$. Let $L_{1}=L$ or $L^{\prime}$ as appropriate. If $\mathcal{N}\left(L_{1}\right)$ takes a value $v \leq q-1$ then we may deduce from (5) that $\mathcal{N}\left(L^{*}\right) \geq v$ for every affine $d$-plane $L^{*}$ parallel to $L_{1}$. Covering $\mathbb{A}^{n}\left(\mathbb{F}_{q}\right)$ with such linear spaces we deduce that $\mathcal{N}\left(\mathbb{A}^{n}\left(\mathbb{F}_{q}\right)\right) \geq v q^{n-d}$. Such bounds as these will suffice in all cases for the theorem.

We begin by establishing part (i) of Theorem 2, From parts (i) and (ii) of Lemma 2 we may obtain a satisfactory bound $\mathcal{N}\left(\mathbb{A}^{n}\left(\mathbb{F}_{q}\right)\right) \geq q^{n+1-d}$ unless either $q=2$ and there is a 2-plane $L_{0} \subseteq \mathbb{A}^{n}\left(\mathbb{F}_{q}\right)$ with $\mathcal{N}\left(L_{0}\right)=3$, or $q \geq 3$ and there is a line $L_{0} \subseteq \mathbb{A}^{n}\left(\mathbb{F}_{q}\right)$ with $2 \leq \mathcal{N}\left(L_{0}\right) \leq q-1$. We now apply Lemma 1. As above we must have $k \leq d$. Moreover if $k \leq d-1$ then (8) yields

$$
\mathcal{N}\left(\mathbb{A}^{n}\left(\mathbb{F}_{q}\right)\right) \geq \frac{q^{n+1-d}-1}{q-1}>q^{n-d}
$$


which is satisfactory. If $k=d$ and $q=2$ then (8) produces

$$
\mathcal{N}\left(\mathbb{A}^{n}\left(\mathbb{F}_{q}\right)\right) \geq 3+\frac{2^{n-d}-1}{2-1}>2^{n-d} .
$$

Finally, if $k=d$ and $q \geq 3$ then $2 \leq \mathcal{N}\left(L_{0}\right)=\mathcal{N}(L) \leq q-1$, and the argument above, with $2 \leq v \leq q-1$, shows that $\mathcal{N}\left(\mathbb{A}^{n}\left(\mathbb{F}_{q}\right)\right) \geq 2 q^{n-d}$. Thus $\mathcal{N}\left(\mathbb{A}^{n}\left(\mathbb{F}_{q}\right)\right)>q^{n-d}$ in all cases.

We turn now to part (ii) of Theorem 2, By part (iii) of Lemma 2 we have $\mathcal{N}\left(\mathbb{A}^{n}\left(\mathbb{F}_{q}\right)\right) \geq q^{n+1-d}-q^{n-d} \geq 2 q^{n-d}$ unless there is a line $L_{0} \subseteq \mathbb{A}^{n}\left(\mathbb{F}_{q}\right)$ with $2 \leq \mathcal{N}\left(L_{0}\right) \leq q-2$. As above, when we apply Lemma 1 we may assume that $k=d-1$ or $d$. If $k=d$ then $2 \leq \mathcal{N}(L) \leq q-2$ and we may apply (5) as before to conclude that $\mathcal{N}\left(\mathbb{A}^{n}\left(\mathbb{F}_{q}\right)\right) \geq 2 q^{n-d}$. Similarly if $k=d-1$ and $\mathcal{N}\left(L^{\prime}\right)-\mathcal{N}(L)=1$ we have $3 \leq \mathcal{N}\left(L^{\prime}\right) \leq q-1$ and therefore $\mathcal{N}\left(\mathbb{A}^{n}\left(\mathbb{F}_{q}\right)\right) \geq 3 q^{n-d}$. Finally, if $k=d-1$ and $\mathcal{N}\left(L^{\prime}\right)-\mathcal{N}(L) \geq 2$ we deduce from (8) that

$$
\mathcal{N}\left(\mathbb{A}^{n}\left(\mathbb{F}_{q}\right)\right) \geq 2 \frac{q^{n+1-d}-1}{q-1} \geq 2 q^{n-d} .
$$

This establishes the required bound in all cases.

To prove the third claim of Theorem 2 we apply Lemma 2 part (iv) with an integer $m \leq q-1$ to be chosen in due course. Thus

$$
\mathcal{N}\left(\mathbb{A}^{n}\left(\mathbb{F}_{q}\right)\right) \geq \frac{m^{n+2-d}-1}{m-1} \geq m^{n+1-d}
$$

unless there is a line with $2 \leq \mathcal{N}\left(L_{0}\right) \leq m$. In the latter case we deduce from Lemma 1 that

$$
\mathcal{N}\left(\mathbb{A}^{n}\left(\mathbb{F}_{q}\right)\right) \geq \frac{q^{n-k}-1}{q-1}\left(\mathcal{N}\left(L^{\prime}\right)-m\right),
$$

and by the previous argument it suffices to consider the values $k=d-1$ and $k=d$. If $k=d-1$ we deduce that $\mathcal{N}\left(\mathbb{A}^{n}\left(\mathbb{F}_{q}\right)\right) \geq q^{n-d}(q-m)$, unless

$$
m+1 \leq \mathcal{N}\left(L^{\prime}\right) \leq q-1
$$

However, in the latter case we conclude by our standard argument using (5) that $\mathcal{N}\left(\mathbb{A}^{n}\left(\mathbb{F}_{q}\right)\right) \geq(m+1) q^{n-d}$. In the homogeneous case the value $k=d$ cannot occur. To show this we consider two cases. If $k=d$ and $\mathbf{0} \in L$ then $q-1 \mid \mathcal{N}(L)-1$, since if $\mathbf{x} \in z(L)$ then every scalar multiple of $\mathbf{x}$ is also in $z(L)$. This however is impossible since $2 \leq \mathcal{N}\left(L_{0}\right)=\mathcal{N}(L) \leq m$. On the other hand, if $\mathbf{0} \notin L$ we consider the $(d+1)$-dimensional linear space $L^{\prime}:=\langle L, \mathbf{0}\rangle$. Here we find that $\mathcal{N}\left(L^{\prime}\right)=1+(q-1) \mathcal{N}(L)$. According to (44) 
we will have $q \mid \mathcal{N}\left(L^{\prime}\right)$, whence $\mathcal{N}(L) \equiv 1(\bmod q)$. This again is impossible, since $2 \leq \mathcal{N}(L) \leq m$. It follows that one of the inequalities

$$
\mathcal{N}\left(\mathbb{A}^{n}\left(\mathbb{F}_{q}\right)\right) \geq m^{n+1-d},
$$

or

$$
\mathcal{N}\left(\mathbb{A}^{n}\left(\mathbb{F}_{q}\right)\right) \geq q^{n-d}(q-m)
$$

or

$$
\mathcal{N}\left(\mathbb{A}^{n}\left(\mathbb{F}_{q}\right)\right) \geq(m+1) q^{n-d}
$$

must hold. The required estimate now follows on choosing

$$
m=\left[\frac{q(n+1-d)}{n+2-d}\right]
$$

and noting that

$$
\left[\frac{q v}{v+1}\right]^{v} \geq \frac{q^{v}}{v+1}
$$

for positive integers $v \geq 2$ and $q \geq 2 v+3$ with the exception of $v=2, q=7$. Since $q-1 \mid \mathcal{N}\left(\mathbb{A}^{n}\left(\mathbb{F}_{q}\right)\right)-1$, the remaining cases follow from parts (i) and (ii) of the theorem, together with (4).

We now prove Lemmas 1 and 2. For the first of these it is enough to observe that $\mathbb{A}^{n}\left(\mathbb{F}_{q}\right)$ is the disjoint union of $L$ together with the sets $L^{*} \backslash L$, with $L^{*}$ running over all $(k+1)$-dimensional linear spaces containing $L$. There are $\left(q^{n-k}-1\right) /(q-1)$ such spaces $L^{*}$. Thus the lemma follows from the fact that $\mathcal{N}\left(L^{*} \backslash L\right) \geq \mathcal{N}\left(L^{\prime}\right)-\mathcal{N}(L)$.

Lemma 2 will require distinctly more work. We prove the four claims separately, using induction on $t$ in each case. The various statements are all trivially true for $t=0$ and $t=1$. To handle the induction step for case (i) we may assume that $S$ contains a linear space $L_{0}$ say, of dimension $t-1$, together with a point $P_{0}$ say, not on $L_{0}$. Now choose any point $P \notin L_{0}$ with $P \neq P_{0}$, and aim to show that $P \in S$. We will then be able to conclude that $S=\mathbb{A}^{t}\left(\mathbb{F}_{q}\right)$ as required. Let $P_{1}$ be any point in $L_{0}$ and consider the 2-plane generated by $P, P_{0}$ and $P_{1}$. Since $q=2$, this plane consists of the three generators together with a fourth point $P_{2}$ say, which must belong to $L_{0}$. Our construction has ensured that $P_{0}, P_{1}$ and $P_{2}$ are all in $S$, and so by our hypothesis we also have $P \in S$, as required.

For part (ii) we use the same initial setup. We assume that $S$ contains a linear space $L_{0}$ of dimension $t-1$, along with a point $P_{0}$ not in $L_{0}$, and we choose a point $P \notin L_{0}$ different from $P_{0}$. Suppose firstly that the line $\ell$ generated by $P_{0}$ and $P$ meets $L_{0}$, at a point $P_{1}$ say. Then $\ell$ meets $S$ in at 
least 2 points, namely $P_{0}$ and $P_{1}$. Then, by our hypothesis, $\ell$ is contained in $S$, whence $P$ in particular belongs to $S$. This deals with all points $P$ except those which lie on the hyperplane, $L_{1}$ say, which is parallel to $L_{0}$ and which passes through $P_{0}$. To handle such points $P$ we begin by fixing any point $P_{1}$ on $L_{0}$. We then consider the line $\ell$ generated by $P$ and $P_{1}$. Since $q \geq 3$ this line contains at least one point additional point $P_{2}$ say, which cannot lie in $L_{1}$. Thus $P_{1} \in S$ by what has already been proved. Hence $\ell$ meets $S$ in at least two points, namely $P_{1}$ and $P_{2}$, and our hypothesis implies that every point of $\ell$ belongs to $S$. In particular $P \in S$, as required.

The proof of part (iii) of Lemma 2 is the most involved. We will write $S^{c}$ for the complement $\mathbb{A}^{t}\left(\mathbb{F}_{q}\right) \backslash S$ of $S$. Our strategy will be to show that if $S^{c}$ also contains $t+1$ points in general position, then both $\# S>\frac{1}{2} q^{t}$ and $\# S^{c}>\frac{1}{2} q^{t}$, which will provide a contradiction. We observe that the hypothesis of part (iii) is symmetric between $S$ and $S^{c}$, since $S$ meets $\ell$ in at least two points if and only if $\#\left(S^{c} \cap \ell\right)<q-1$. Now let $R$ be either $S$ or $S^{c}$, and assume that $R$ contains $t+1$ points $P_{0}, \ldots, P_{t}$ in general position. For our inductive assumption we suppose that, for any linear space $L \subset \mathbb{A}^{t}\left(\mathbb{F}_{q}\right)$ of dimension $t-1$, either $R \cap L$ fails to contain $t$ points in general position, or $L \backslash R$ is contained in a proper linear subspace of $L$. Thus either $L \cap R$ or $L \cap R^{c}$ lies in a proper linear subspace of $L$. When $L=L_{0}$ is generated by $P_{1}, \ldots, P_{t}$ we must be in the second case. For every $P \in L_{0}$, the line $\ell$ generated by $P$ and $P_{0}$ meets $R$ in at least two points (namely $P$ and $P_{0}$ ) and hence contains at least $q-1$ points of $R$. For distinct choices of $P$ the sets $\ell \backslash\left\{P_{0}\right\}$ are disjoint, whence

$$
\# R \geq 1+(q-2) \#\left(L_{0} \cap R\right) .
$$

Now suppose that every linear space $L$ of dimension $t-1$, parallel but not equal to $L_{0}$, has the property that $L \cap R$ lies in a proper linear subspace of $L$. Then since $\mathbb{A}^{t}\left(\mathbb{F}_{q}\right)$ is a disjoint union of $L_{0}$ with the various spaces $L$, we see that

$$
\# R \leq \#\left(L_{0} \cap R\right)+(q-1) q^{t-2} .
$$

Comparing this with (9) yields $(q-3) \#\left(L_{0} \cap R\right)<(q-1) q^{t-2}$, and since $\#\left(L_{0} \cap R\right) \geq q^{t-1}-q^{t-2}$ we find that $q<4$. Thus, under the assumption that $q \geq 4$, there must be at least one space $L_{1}$, parallel but not equal to $L_{0}$, for which it is $L_{1} \cap R^{c}$ which is contained in a proper linear subspace of $L_{1}$. If we pick any point $Q \in L_{1}$ and count points of $R$ on lines from $Q$ to $L_{0}$ we will obtain at least $(q-2) \#\left(L_{0} \cap R\right)$ points of $R$ not lying on $L_{1}$, by the argument that established (9). Allowing for points of $L_{1} \cap R$ we find that

$$
\# R \geq(q-2) \#\left(L_{0} \cap R\right)+\#\left(L_{1} \cap R\right) .
$$


However we have arranged that $L_{0} \cap R^{c}$ and $L_{1} \cap R^{c}$ are both contained in proper linear subspaces, so that $\#\left(L_{0} \cap R\right) \geq q^{t-1}-q^{t-2}$, and similarly for $\#\left(L_{1} \cap R\right)$. It then follows that $\# R \geq(q-1)^{2} q^{t-2}>\frac{1}{2} q^{t}$, since $q \geq 4$. As explained above, this inequality leads to the claim made in part (iii) of the lemma.

Finally we turn to part (iv) of Lemma 2, For the induction step we assume that we have a linear space $L_{0}$ of dimension $t-1$, along with a point $P_{0}$ not in $L_{0}$, such that

$$
\#\left(S \cap L_{0}\right) \geq \frac{m^{t}-1}{m-1}
$$

and $P_{0} \in S$. If $P$ is any point in $S \cap L_{0}$ the line $\ell$ generated by $P$ and $P_{0}$ contains at least 2 points of $S$, and hence by our hypothesis contains at least $m+1$ such points. For different points $P$ the sets $\ell \backslash\left\{P_{0}\right\}$ are disjoint, whence

$$
\# S \geq 1+m \#\left(S \cap L_{0}\right) \geq 1+m \frac{m^{t}-1}{m-1}=\frac{m^{t+1}-1}{m-1} .
$$

This completes the induction.

We now establish Theorem 3. If any of the polynomials $\mathbf{f}$ vanishes identically we may clearly remove it from the collection, and if any of the polynomials is a non-zero constant then $z\left(\mathbf{f} ; \mathbb{A}^{n}\left(\mathbb{F}_{q}\right)\right)$ is empty, so that the result is trivial. It follows that we may assume that each of the $f_{i}$ has positive degree, whence $r$, which is the number of polynomials $f_{i}$, is at most $d$. Thus the zero-set of the polynomials $\mathbf{f}$, over $\overline{\mathbb{F}_{q}}$ is an algebraic variety, $V$ say, of dimension at most $n$. This variety is defined over $\mathbb{F}_{q}$, but is not necessarily absolutely irreducible. We shall call an irreducible component of $V$ "bad" if it is not defined over $\mathbb{F}_{q}$. Thus our goal is to replace $V$ by a variety $V^{*}$ with no bad components, and such that $V\left(\mathbb{F}_{q}\right)=V^{*}\left(\mathbb{F}_{q}\right)$. We proceed to describe a general "reduction process" for our varieties. In order to keep track of the number of absolutely irreducible components that these varieties have, and of their degrees, it will be convenient to define $\delta(X)=\sum \operatorname{deg}\left(X_{i}\right)$ whenever the variety $X$ is a union of absolutely irreducible components $X_{i}$. It follows that $\delta(V) \leq C_{0}(n, d)$ for some number $C_{0}(n, d)$ depending on $n$ and $d$ alone, and we aim to establish an analogous bound $\delta\left(V^{*}\right) \leq C(n, d)$ for the variety $V^{*}$.

Suppose that $V$ has at least one bad component. Choose such a component, $W$ say, of maximal dimension $D$ say. Let the Galois conjugates of $W$ be $W=W_{1}, \ldots, W_{k}$. Since each of these is an irreducible component of $V$ we see that $k \leq C_{0}(n, d)$. Any $\mathbb{F}_{q}$-point of $W$ lies on $W^{\prime}:=W_{1} \cap \ldots \cap W_{k}$. Thus if we replace the components $W_{1}, \ldots, W_{k}$ of $V$ by $W^{\prime}$, and call the 
resulting variety $V_{1}$, we see that $V\left(\mathbb{F}_{q}\right)=V_{1}\left(\mathbb{F}_{q}\right)$. Moreover $V_{1}$ contains no bad components of dimension greater than $D$, and since $\operatorname{dim}\left(W^{\prime}\right)<\operatorname{dim}(W)$ there is one fewer bad component of dimension $D$. We also note that $\delta\left(W^{\prime}\right)$ can be bounded in terms of $n$ and $C_{0}(n, d)$ alone whence $\delta\left(V_{1}\right) \leq C_{1}(n, d)$ for a suitable integer $C_{1}(n, d)$. We now repeat the reduction process, passing from $V_{1}$ to $V_{2}$ and so on. Since the number of bad components of maximal dimension is reduced at each step, while $\delta\left(V_{i}\right)$ remains under control, the process will eventually terminate, and will produce a variety $V^{*}$ for which $\delta\left(V^{*}\right)$ is bounded in terms of $n$ and $d$. The theorem then follows.

\section{References}

[1] J. Ax, Zeros of polynomials over finite fields, Amer. J. Math., 86 (1964), 255-261.

[2] C. Chevalley, Démonstration d'une hypothèse de M. Artin, Abh. Math. Sem. Univ. Hamburg, 11 (1935), 73-75.

[3] E. Warning, Bemerkung zur vorstehenden Arbeit von Herrn Chevalley, Abh. Math. Sem. Univ. Hamburg, 11 (1935), 76-83.

[4] N.M. Katz, On a theorem of Ax, Amer. J. Math., 93 (1971), 485-499.

[5] S. Lang and A. Weil, Number of points of varieties in finite fields, Amer. J. Math., 76 (1954), 819-827.

Mathematical Institute,

Radcliffe Observatory Quarter

Woodstock Road

Oxford

OX2 6GG

$\mathrm{UK}$

rhb@maths.ox.ac.uk 